DFTUZ/96-14

hep-th/9605455

\title{
Effective Field Theory of Gravity, Reduction of Couplings and the Renormalization Group
}

\author{
Mario Atance*网 and José Luis Cortés河 \\ Departamento de Física Teórica, \\ Universidad de Zaragoza, 50009 Zaragoza, Spain.
}

May 30, 1996

\begin{abstract}
The structure of the renormalization group equations for the low energy effective theory of gravity coupled to a scalar field is presented. An approximate solution to these equations with a finite number of independent renormalized parameters can be found when the mass scale characteristic of the fluctuations in the geometry is much smaller than the Planck mass. The cosmological constant problem is reformulated in this context and some conditions on the matter field content and interactions required in order to have a sufficiently small cosmological constant are identified.
\end{abstract}

*E-mail atance@posta.unizar.es

†E-mail cortes@posta.unizar.es 


\section{INTRODUCTION}

This work can be classified within the perturbative approach in which quantum gravity is seen as a theory of small quantum fluctuations around a flat Minkowski background spacetime. From this point of view quantum gravity can be regarded as just another field theory to be quantised in a standard way as it should be the case for any relativistic quantum theory at low energy [1]. This leads to identify an effective field theory with the gravitational field described by a symmetric two-index tensor field as the low energy effective field theoretic formulation of quantum gravity.

Like any effective field theory its Lagrangian density will contain an infinite number of terms of arbitrary dimensionality and, therefore, is not perturbatively renormalizable in the usual power counting sense [2]. But it is renormalizable in the sense that all the ultraviolet divergences can be cancelled by a renormalization of the infinite number of parameters corresponding to the most general action invariant under general covariant transformations [3]. Then the perturbative approach of renormalized effective theories [4] can also be applied in general relativity. This point of view has been recently advocated by J.F.Donoghue [5] who shows how some large distance quantum gravitational effects can be derived within this framework.

In order to compute results for physical quantities in an effective field theory it is necessary to specify the lagrangian together with a renormalization scheme. A natural way to parametrize the lagrangian is based on the introduction of a fixed mass scale $M$, which is a characteristic scale of the physical system described by the effective theory, and a dimensionless parameter for each term in the lagrangian giving the corresponding coefficient in units of $M$ raised to the appropriate power. Then one has an expansion of the lagrangian with terms of dimensionality greater than four suppressed by negative powers of the mass scale $M$. If one wants to have a well-defined expansion (with terms of higher dimension being 
less important in the calculation of physical quantities) a mass-independent renormalization scheme must be chosen [6].

Each term in the lagrangian of the effective theory of gravity is a product of (covariant derivatives of) matter fields and components of the Riemann tensor. At sufficiently low energies and for sufficiently small fluctuations in the matter fields and the geometry of space-time, the lower dimensional terms in the lagrangian will be dominant and the expansion in the effective lagrangian is a good approximation. At higher energies, and/or for larger fluctuations of the matter system or the geometry, higher order terms in the effective lagrangian become comparable to the lower dimensional terms. There is an intermediate situation where corrections to the dominant term can be incorporated as a small perturbation.

In the general case, at each order in the expansion new free parameters appear and the predictibility of the effective theory is reduced. But this is not always necessarily the case. The renormalization group equations, fixing the dependence of the renormalized parameters on the renormalization scale, allow to identify special situations where only a finite number of renormalized parameters can be chosen freely. In those cases the predictibility of the theory is not lost when successive terms in the expansion of the effective theory are incorporated.

If one goes beyond the domain of validity of the perturbative approach to gravity then new interactions and new degrees of freedom will be required in a new theory beyond quantum field theory based on some unknown general principles [7]. The main point of this work is to investigate the possibility that this new theory going beyond the perturbative regime is such that its low energy limit is as independent on the details of the theory as possible. In other words we consider an effective field theory with a minimal number of free renormalized parameters.

In a previous work [8] the general structure of the renormalization group equations for the effective field theory of pure gravity was identified. In the limiting case where the mass scale of the effective theory is much smaller than the Planck mass (a possibility compatible with the renormalization group equations), a theory with just one free renormalized parameter is 
obtained when contributions suppressed by inverse powers of the Planck mass are neglected. The aim of this work is to extend these results to the effective field theory of gravity, including matter fields and non-gravitational interactions. Additional renormalization group equations for the new parameters as well as the modifications induced by these new parameters on the renormalization group equations of the pure gravity theory have to be considered. Then one has to identify what are the conditions to be able to express the effective lagrangian in terms of a finite number of free parameters in a way consistent with the renormalization group equations.

In the next section we consider the renormalization of the theory of a scalar field coupled to a symmetric two-index tensor field invariant under general covariant transformations. The absence of a dimensionfull ultraviolet cut-off in a mass independent substraction scheme (dimensional regularization and minimal substraction) allows us to give the general structure of the renormalization group equations for the dimensionless renormalized parameters of the theory. The renormalized parameters corresponding to terms in the lagrangian of dimension less than four (mass and cosmological term) can be set equal to zero. In this case the renormalization group equations for the remaining parameters have a triangular structure. For a given term in the lagrangian, the renormalization group equation for the corresponding parameter depends only on a finite number of parameters corresponding to the terms in the lagrangian of dimensionality smaller or equal than that of the original term. The $\beta$ functions are fixed by dimensional arguments up to the dependence on the parameter corresponding to the scalar quartic self-coupling term. There is a series expansion in this parameter which can be determined order by order in perturbation theory.

In section 3 a discussion of the possibility to find a renormalized lagrangian with a finite number of independent renormalized parameters is presented. Two special cases are identified. In the first case one has a generalization of the reduction obtained in a previous work in pure gravity [8] with a mass scale $M_{R}$, characteristic of the fluctuations in the geometry of space-time, much smaller than the Planck mass and an additional dependence on the scalar self-coupling determined perturbatively. A second case corresponds to the 
presence of still another new independent parameter corresponding to a term of dimension six in the matter field lagrangian. This new parameter defines a new mass scale, associated to the matter field fluctuations, together with the Planck mass and the scale $M_{R}$. In both cases the reduction of the infinite number of parameters in the general effective lagrangian in terms of three or four independent parameters can be determined systematically order by order in perturbation theory by using the renormalization group equations of the effective theory.

In section 4 we discuss the modification induced in the renormalization group equations when a mass term in the lagrangian is added. In the general case a cosmological term is unavoidable and, due to the presence of terms of dimension less than four in the lagrangian, the renormalization group equations lose its simple structure and all the infinite parameters appear in the renormalization group equation of each parameter. The only way to translate to this case the discussion of the possibility to have an effective theory with a finite number of parameters is based on the assumption that the dimensionless parameters corresponding to the mass and cosmological terms in the lagrangian are sufficiently small to treat the modifications they induce on the $\beta$ functions of the remaining parameters as a small perturbation that can be neglected in a first approximation. In fact the experimental upper bound on the cosmological constant forces this to be the case for the cosmological term and the parameter corresponding to the mass term has to be tuned in such a way that the scalar mass is much smaller than the remaining mass scales of the effective theory. The consistency of the tuning required on the cosmological parameter with its renormalization group equation leads to a reformulation of the cosmological constant problem [9] at the level of the effective theory. Different alternatives to the solution of this problem in connection with the reduction of parameters in the effective theory of gravity are discussed.

We end up in section 5 with a summary and some concluding comments. 


\section{RENORMALIZATION GROUP EQUATIONS. MASSLESS CASE}

The main idea in this work is to try to get some information on quantum gravity from the renormalization group equations of its effective field theory formulation. This equations can be derived following the same steps of a perturbatively renormalizable theory in the power counting sense [10]. A very important tool in the perturbative renormalization of an effective field theory is dimensional regularization [11] and the minimal substraction scheme [12].

We will consider for definiteness the simplest matter system (a real scalar field) coupled to the gravitational field but most of the discussion can be translated directly to a general matter system. It is convenient to introduce a fixed mass scale $M$ as a reference unit for all the couplings of the effective theory. The general expression for the action can be written, using the invariance under general covariant transformations, in the following form

$$
S=\int d^{4} x\left[\mathcal{L}_{g}+\mathcal{L}_{m}+\mathcal{L}_{n . m .}\right]
$$

where $\mathcal{L}_{g}$, is the lagrangian density of the gravitational field, and $\mathcal{L}_{m}, \mathcal{L}_{n . m}$. are the lagrangian densities for the minimal and non-minimal coupling of the scalar and gravitational fields respectively

$$
\begin{gathered}
\mathcal{L}_{g}=\sqrt{-g} \frac{M^{2}}{\alpha_{1}^{2}}\left[R+\frac{\vec{\alpha}_{2}}{M^{2}} \vec{R}^{(2)}+\frac{\vec{\alpha}_{4}}{M^{4}} \vec{R}^{(4)}+\ldots\right], \\
\mathcal{L}_{m}=\sqrt{-g}\left[\frac{1}{2} g^{\mu \nu} \partial_{\mu} \phi \partial_{\nu} \phi+\lambda_{0} \phi^{4}+\frac{\vec{\lambda}_{2}}{M^{2}} \overrightarrow{\mathcal{L}}_{m}^{(2)}+\frac{\vec{\lambda}_{4}}{M^{4}} \overrightarrow{\mathcal{L}}_{m}^{(4)}+\ldots\right], \\
\mathcal{L}_{n . m .}=\sqrt{-g}\left[\xi_{0} R \phi^{2}+\frac{\vec{\xi}_{2}}{M^{2}} \overrightarrow{\mathcal{L}}_{n . m .}^{(2)}+\frac{\vec{\xi}_{4}}{M^{4}} \overrightarrow{\mathcal{L}}_{n . m .}^{(4)}+\ldots\right] .
\end{gathered}
$$

A cosmological constant (constant term inside the brackets in (2.2) ) and a mass $\left(\phi^{2}\right.$ term in $(2.3)$ ) have not been included (later we will see how the structure of the renormalization group equations would be affected in the presence of such terms). The coefficients $\alpha_{1}, \vec{\alpha}_{2}$, $\vec{\alpha}_{4}, \ldots, \lambda_{0}, \vec{\lambda}_{2}, \vec{\lambda}_{4}, \ldots, \xi_{0}, \vec{\xi}_{2}, \vec{\xi}_{4}, \ldots$, are dimensionless parameters.

The dimensionality of the different terms in the action fixes the power dependence on the mass scale $M$ of the effective theory. In the lagrangian density of pure gravity $\mathcal{L}_{g}, R$ 
is the scalar curvature, $\vec{R}^{(2)}$ is a vector with the three different invariants built out of two Riemann tensors as components, the different invariants with three Riemann tensors or two Riemann tensors and two derivatives are the components of the vector $\vec{R}^{(4)}$ and so on. The expansion in inverse powers of the mass scale for the terms depending on the matter field has been written in a compact notation where $\overrightarrow{\mathcal{L}}_{m}^{(2 n)}$ is a vector whose components are the different terms of dimension $4+2 n$ built out of the scalar field and derivatives of the scalar field with all derivatives replaced by general covariant derivatives. The additional terms of the same dimension involving the Riemann tensor are the components of $\overrightarrow{\mathcal{L}}_{n . m}^{(2 n)}$ in the energy expansion of the nonminimal coupling of the scalar and gravitational fields (2.4).

For the first terms in the effective field theory expansion one has

$$
\begin{aligned}
\vec{R}^{(2)} & =\left(R^{2}, R_{\mu \nu} R^{\mu \nu}, \epsilon^{\alpha \beta \gamma \delta} \epsilon_{\mu \nu \rho \sigma} R^{\mu \nu}{ }_{\alpha \beta} R^{\rho \sigma}{ }_{\gamma \delta}\right) \\
\overrightarrow{\mathcal{L}}_{m}^{(2)} & =\left(\left(g^{\mu \nu} D_{\mu} \partial_{\nu} \phi\right)^{2}, \phi^{2} g^{\mu \nu} \partial_{\mu} \phi \partial_{\nu} \phi, \phi^{6}\right) \\
\overrightarrow{\mathcal{L}}_{n . m .}^{(2)} & =\left(\vec{R}^{(2)} \phi^{2}, R g^{\mu \nu} \partial_{\mu} \phi \partial_{\nu} \phi, R^{\mu \nu} \partial_{\mu} \phi \partial_{\nu} \phi, R \phi^{4}\right) .
\end{aligned}
$$

The general parametrization of the effective action is redundant for two different reasons. First, a change in the scale $M$ is equivalent to an appropriate rescaling of every dimensionless parameter. Second, by using a non-linear redefinition of fields it is possible to eliminate some of the terms in the action (2.1)-(2.4). Nevertheless it is simpler to use this redundant parametrization in order to identify the general structure of the renormalization group equations. There are two simple examples for a convenient choice of the mass scale of the effective theory $M$. If there is a choice of the scale $M$ such that all the dimensionless parameters $\alpha_{2 n}^{(i)}$ in the lagrangian density of the gravitational field $\mathcal{L}_{g}$ are simultaneously of order one then this scale characterizes the size of the fluctuations in the geometry. Alternatively, if there is a choice of $M$ such that all the dimensionless parameters $\lambda_{2 n}^{(i)}, \xi_{2 n}^{(i)}$ are of order one then this is the scale characteristic of the matter field fluctuations. Once the scale of the effective theory $M$ has been choosen as one of the scales of the physical system, using the Newtonian limit of this action one has $\alpha_{1}^{2}=16 \pi\left(\frac{M}{M_{P l}}\right)^{2}$ where $M_{P l}$ is the Planck mass. Then $\alpha_{1}$ gives the scale $M$ in units of the Planck mass. 
The simplest case, a system with a unique natural scale (Planck mass), corresponds to an effective action with all the dimensionless parameters of order one when $M \sim M_{P l}$. Next one can consider a case where all the parameters except $\alpha_{1}$ are of order one at a given value of $M$ and it corresponds to a system with two mass scales, one associated to the classical limit and a common scale for the geometry and matter field fluctuations. Another possibility is that there are two different choices for $M$, one making all $\alpha_{2 n}^{(i)}$ of order one and a second one making all $\lambda_{2 n}^{(i)}, \xi_{2 n}^{(i)}$ of order one; in this case there are two different scales for the fluctuations of the geometry and the matter system together with the Planck scale. One could consider even more complicated systems with more and more different characteristic mass scales corresponding at the level of the effective field theory to richer hierarchies for the dimensionless parameters.

A perturbative analysis of the action (2.1)-(2.4) based on the decomposition of the metric

$$
g_{\mu \nu}=\eta_{\mu \nu}+\frac{\alpha_{1}}{M} h_{\mu \nu} \quad \eta_{\mu \nu}=\operatorname{diag}(1,-1,-1,-1),
$$

can be done [13 [15] by using the standard methods of gauge theories. A non-invariant term (gauge fixing) has to be added to the action (2.1), for example

$$
S_{g . f .}=\int d^{4} x \sqrt{-g} \frac{1}{2}\left(\partial^{\rho} h_{\mu \rho}-\frac{1}{2} \partial_{\mu} h_{\rho}^{\rho}\right)^{2}
$$

is a very convenient choice for explicit calculations. The standard derivation, in perturbatively renormalizable theories, of the renormalization group in a mass independent renormalization scheme [10] can be translated to an effective field theory. One has an infinite number of bare parameters in one to one correspondence with the dimensionless parameters of the effective action. Using dimensional regularization one has expressions for the bare parameters in terms of the renormalized parameters with poles when $\epsilon \rightarrow 0$ (dimension $D=4-\epsilon)$. From the independence of the bare parameters on the renormalization scale $\mu$, one concludes that any change of $\mu$ must be equivalent to a change in the renormalized parameters. The renormalization group equations express this fact.

In the case of gravity coupled to a scalar field these equations are 


$$
\begin{gathered}
\mu \frac{d \alpha_{1}}{d \mu}=\beta_{\alpha_{1}}\left(\vec{\alpha}, \vec{\lambda}, \alpha_{1} \vec{\xi}\right), \mu \frac{d \alpha_{2 n}^{(i)}}{d \mu}=\beta_{\alpha_{2 n}^{(i)}}\left(\vec{\alpha}, \vec{\lambda}, \alpha_{1} \vec{\xi}\right) \\
\mu \frac{d \lambda_{2 n}^{(i)}}{d \mu}=\beta_{\lambda_{2 n}^{(i)}}\left(\vec{\alpha}, \vec{\lambda}, \alpha_{1} \vec{\xi}\right) \\
\mu \frac{d\left(\alpha_{1} \xi_{2 n}^{(i)}\right)}{d \mu}=\beta_{\alpha_{1} \xi_{2 n}^{(i)}\left(\vec{\alpha}, \vec{\lambda}, \alpha_{1} \vec{\xi}\right)}
\end{gathered}
$$

where $\vec{\alpha}, \vec{\lambda}, \vec{\xi}$, are the set of dimensionless parameters appearing in $\mathcal{L}_{g}, \mathcal{L}_{m}, \mathcal{L}_{n . m}$. respectively. The renormalization group $\beta$ functions are determined perturbatively from the residues of the simple poles at $\epsilon=0$ in the relations between bare and renormalized dimensionless parameters. In the parametrization used in (2.1)-(2.4) any interaction term with the gravitational field $h_{\mu \nu}$ is proportional to $\alpha_{1}$. This is the reason why the parameters corresponding to the non-minimal coupling appear in the renormalization group equations through the combination $\alpha_{1} \vec{\xi}$.

Dimensional arguments together with the presence of a single mass scale $M$ ( the dependence on the renormalization scale $\mu$ is logarithmic ) lead to identify a simple structure for the renormalization group equations which is a generalization of the structure found in the case of pure gravity [8]. The $\beta$ functions satisfy the homogeneity conditions

$$
\begin{gathered}
\beta_{\alpha_{1}}\left(\vec{\alpha}^{\prime}, \vec{\lambda}^{\prime}, \alpha_{1}^{\prime} \vec{\xi}^{\prime}\right)=t \beta_{\alpha_{1}}\left(\vec{\alpha}, \vec{\lambda}, \alpha_{1} \vec{\xi}\right), \\
\beta_{\alpha_{2 n}^{(i)}}\left(\vec{\alpha}^{\prime}, \vec{\lambda}^{\prime}, \alpha_{1}^{\prime} \vec{\xi}^{\prime}\right)=t^{2 n} \beta_{\alpha_{2 n}^{(i)}}\left(\vec{\alpha}, \vec{\lambda}, \alpha_{1} \vec{\xi}\right), \\
\beta_{\lambda_{2 n}^{(i)}}\left(\vec{\alpha}^{\prime}, \vec{\lambda}^{\prime}, \alpha_{1}^{\prime} \vec{\xi}^{\prime}\right)=t^{2 n} \beta_{\lambda_{2 n}^{(i)}}\left(\vec{\alpha}, \vec{\lambda}, \alpha_{1} \vec{\xi}\right), \\
\beta_{\alpha_{1} \xi_{2 n}^{(i)}}\left(\vec{\alpha}^{\prime}, \vec{\lambda}^{\prime}, \alpha_{1}^{\prime} \vec{\xi}^{\prime}\right)=t^{2 n+1} \beta_{\alpha_{1} \xi_{2 n}^{(i)}}\left(\vec{\alpha}, \vec{\lambda}, \alpha_{1} \vec{\xi}\right) .
\end{gathered}
$$

where

$$
\alpha_{1}^{\prime}=t \alpha_{1}, \quad \vec{\alpha}_{2 n}^{\prime}=t^{2 n} \vec{\alpha}_{2 n}, \vec{\lambda}_{2 n}^{\prime}=t^{2 n} \vec{\lambda}_{2 n}, \vec{\xi}_{2 n}^{\prime}=t^{2 n} \vec{\xi}_{2 n}
$$


These conditions put strong restrictions on the dependence of the $\beta$ functions on all the dimensionless parameters with one exception, the scalar self coupling $\lambda_{0}$. Each renormalization group beta function will be a polinomial in the remaining parameters of a given degree with a series expansion in $\lambda_{0}$ as coefficients which can be determined order by order in perturbation theory. From (2.12)-(2.14) one can see that the $\mu$ dependence of $\vec{\alpha}_{2 n}, \vec{\lambda}_{2 n}$, $\vec{\xi}_{2 n}$, is fixed by a finite number of parameters $\vec{\alpha}_{k}, \vec{\lambda}_{k}$ and $\vec{\xi}_{k}$ with $k \leq 2 n$. This triangular structure of the renormalization group equations in a mass independent renormalization scheme is the main ingredient in the discussion of the effective field theory formulation of gravity presented in this work.

A mass for the scalar field and a cosmological constant term corresponds, in the parametrization of the effective field theory we are using, to the addition of a term $\lambda_{-2} M^{2} \phi^{2}$ and $\alpha_{-2} M^{2}$ inside the brackets in (2.3) and (2.2) respectively. The modifications in the renormalization group equations are the addition of two equations for $\alpha_{-2}$ and $\lambda_{-2}$ and the presence of new contributions in the $\beta$ functions depending on $\alpha_{-2}, \lambda_{-2}$. The homogeneity conditions (2.11)-(2.14) can be generalized including $\alpha_{-2}^{\prime}=t^{-2} \alpha_{-2}$ and $\lambda_{-2}^{\prime}=t^{-2} \lambda_{-2}$. One consequence of these conditions is that $\beta_{\alpha_{-2}}$ and $\beta_{\lambda_{-2}}$ are both proportional to $\alpha_{-2}$ or $\lambda_{-2}$ and then a vanishing mass and cosmological constant parameters, required in order to get the simple triangular structure for the $\mu$ dependence of the parameters of the effective action, is consistent with the renormalization group equations.

To illustrate the general previous considerations we will end up this section by writing explicitly the renormalization group equations for the first terms in the $\frac{1}{M}$ expansion of the effective action. One has

$$
\begin{gathered}
\mu \frac{d \lambda_{0}}{d \mu}=\lambda_{0}^{2} B_{0}^{(1)}, \\
\mu \frac{d \xi_{0}}{d \mu}=\lambda_{0} B_{0}^{(2)}\left(1-\frac{\xi_{0}}{12}\right),
\end{gathered}
$$

where $B_{0}^{(k)}$ are power expansions in $\lambda_{0}$. There is no effect of the gravitational interaction on the $\mu$ dependence of the scalar self-coupling and (2.16) is the renormalization group 
equation of the $\lambda \phi^{4}$ theory. The $\beta$ function for $\xi_{0}$ can be read from the insertion of an external graviton and a single gravitational coupling on the scalar self-energy and it does not involve any effect from the fluctuations of the gravitational field. This is the reason why there is a common power expansion in $\lambda_{0}$ for the two terms in the $\beta$ function for $\xi_{0}$, and the relative coefficient, -12 , can be read from the one loop results for the divergencies of gravitation interacting with a scalar particle [13.

Next one has

$$
\mu \frac{d \alpha_{1}}{d \mu}=0
$$

This is because any gravitational interaction is proportional to $\alpha_{1}$ and it is not posssible to have a divergent $\frac{1}{\epsilon}$ contribution compatible with (2.11). At order $\frac{1}{M^{2}}$ the renormalization group equations are

$$
\begin{gathered}
\mu \frac{d \alpha_{2}^{(i)}}{d \mu}=\alpha_{1}^{2}\left[B_{2}^{(i, 1)}+\xi_{0} B_{2}^{(i, 2)}+\xi_{0}^{2} B_{2}^{(i, 3)}\right], \\
\mu \frac{d \lambda_{2}^{(i)}}{d \mu}=\sum_{j} \lambda_{2}^{(j)} \lambda_{0} B_{2}^{(i, j, 4)}+\alpha_{1}{ }^{2} \lambda_{0}\left[B_{2}^{(i, 5)}+\xi_{0} B_{2}^{(i, 6)}+\xi_{0}{ }^{2} B_{2}^{(i, 7)}\right], \\
\mu \frac{d \xi_{2}^{(i)}}{d \mu}=\sum_{j} \xi_{2}^{(j)} \lambda_{0} B_{2}^{(i, j, 8)}+\sum_{j} \lambda_{2}^{j}\left[B_{2}^{(j, 9)}+\xi_{0} B_{2}^{(j, 10)}\right]+ \\
\alpha_{1}{ }^{2}\left[B_{2}^{(i, 11)}+\xi_{0} B_{2}^{(i, 12)}+\xi_{0}{ }^{2} B_{2}^{(i, 13)}+\xi_{0}{ }^{3} B_{2}^{(i, 14)}\right],
\end{gathered}
$$

where once more one has several coefficients $B_{2}$ as power expansions in the self-coupling $\lambda_{0}$. The generalization to higher orders terms in the $\frac{1}{M}$ expansion is obvious.

The zero order term in the expansion of $B_{2}^{(i, 1)}, B_{2}^{(i, 2)}, B_{2}^{(i, 3)}$ for the gauge fixing in (2.7) can be read from the one loop calculations in [13,14. The determination of the renormalization group coefficients for $\alpha_{4}^{(i)}$ in lowest order requires a two loop calculation whose results in the case of pure gravity are given in [14]. Although the divergences, and then the coefficients of the renormalization group equations, depend on the gauge fixing condition [16], there are no additional substractions and a modification of the gauge fixing condition simply gives an equivalent formulation of the effective field theory. 
The general structure of the renormalization group equations based on dimensional arguments will be valid beyond the simple example of a scalar field considered in this section. The only difference will be on the number and explicit form of the different terms in the minimal and non-minimal coupling of the matter system to the gravitational field and on the values for the coefficients of the counterterms which determine the coefficients in the renormalization group equations.

\section{REDUCTION OF COUPLINGS}

\section{A. General considerations}

The idea of looking for relations between the couplings of a renormalizable field theory which are independent of the renormalization scale and compatible with the renormalizability of the theory has been studied in recent years for different purposes [17]. The program of reduction of couplings was initiated in [18] by looking for massless renormalizable theories in the power counting sense with a single dimensionless coupling parameter. The same basic ideas were considered in [19] in order to understand the presence of a finite number

of parameters in a renormalizable theory despite the appearence of an infinite number of interaction terms in the light-cone quantization method. The underlying Lorentz covariance and gauge invariance, which are not manifest in this quantization scheme, are reflected in the possibility to determine the renormalization group trajectories in terms of a finite number of running variables.

In the case of the effective field theory formulation of gravity one also has an infinite number of interaction terms as required by the ultraviolet divergences of the theory but one can consider the possibility to have a finite number of independent renormalized parameters. The reduction of couplings in this case could be a consequence of a symmetry of the underlying fundamental theory which is hidden in the field theoretical limit. If the effective field theory of gravity is a result of the application of the reduction program then one has 
a situation where the field theoretical approach goes as far as possible in the sense that the low energy limit of the theory is only sensitive to the details of the underlying theory through the value of a finite number of parameters.

This idea has been studied in the simpler case of pure gravity in [8]. The result is that it is possible to express all the parameters $\vec{\alpha}_{2 n}$ in terms of two parameters $\alpha_{1}$ and $\alpha_{2}$ in a way compatible with the renormalization group equations. But in the general case there are as many free parameters in these relations as parameters $\vec{\alpha}_{2 n}$ due to the trivial $\mu$ dependence of $\alpha_{1}$. Then one has a real reduction of couplings only when one assumes that $\alpha_{1}^{2} \ll \alpha_{2}$, which corresponds to a mass scale for the fluctuations of the geometry much smaller than the Planck mass, and keeps only the dominant term in the expansion in powers of $\alpha_{1}$. The aim of this section is to consider possible extensions of the reduction of couplings in the presence of a scalar matter field coupled to the gravitational field. The main difference with the pure gravity case is the presence of a parameter, $\lambda_{0}$, in the renormalization group equations and in the relations between couplings. The dependence on this new parameter is not fixed by dimensional considerations and is incorporated order by order in a perturbative expansion. Another difference comes from the possibility to consider new reductions of couplings with an additional independent parameter $\lambda_{2}$ associated to the matter system.

\section{B. Minimal reduction}

The triangular structure of the renormalization group equations allows to look for reduction of parameters order by order in the $\frac{1}{M}$ expansion of the effective theory. In lowest order the relevant parameters to consider are $\lambda_{0}, \xi_{0}$ and the renormalization group equations (2.16), (2.17) - A reduction at this level corresponds to express $\xi_{0}$ as a power expansion in $\lambda_{0}$ with coefficients fixed in order to reproduce its $\mu$ dependence. The only posssible reduction is a trivial one, $\xi_{0}=\frac{1}{12}$.

At order $\frac{1}{M^{2}}$ the first parameter to consider is $\vec{\alpha}_{2}$. The renormalization group equation (2.19) can be written, using the reduction of $\xi_{0}$, as 


$$
\mu \frac{d \vec{\alpha}_{2}}{d \mu}=\vec{A}_{2} \alpha_{1}^{2}
$$

The factorization at this level of the radiative corrections due to the matter field leads to identify a unique power expansion in $\lambda_{0}, A_{2}$,

$$
\vec{A}_{2}=\vec{a}_{2}^{(0)} A_{2}
$$

where the zero order term in $A_{2}$ is chosen to be one and the constant coefficients $\vec{a}_{2}^{(0)}$ can be read from the one loop result [13] for the counterterms quadratic in the Riemann tensor. A reduction of couplings at this level can be obtained if one introduces a dimensionless parameter $\alpha_{2}$ with a renormalization scale dependence given by

$$
\mu \frac{d \alpha_{2}}{d \mu}=A_{2} \alpha_{1}^{2}
$$

Then it is possible to write the parameters $\alpha_{2}^{(i)}$ in terms of $\alpha_{2}$ and $\alpha_{1}$ in a way compatible with the renormalization group equations,

$$
\vec{\alpha}_{2}=\vec{a}_{2}^{(0)} \alpha_{2}+\vec{a}_{2}^{(1)} \alpha_{1}^{2}
$$

but, as a consequence of the independence of $\alpha_{1}$ on the renormalization scale, the coefficients $\vec{a}_{2}^{(1)}$ are free parameters and the relations (3.4) are not real reduction equations but simply a reparametrization. If $\alpha_{1}^{2} \ll \alpha_{2}$, and one takes the dominant term in the expansion in powers of $\frac{\alpha_{1}^{2}}{\alpha_{2}}$ in (3.4), then one has a reduction of couplings for the terms quadratic in the Riemann tensor.

The next step is to consider the renormalization group equations for the parameters corresponding to terms of dimension 6 in the minimal coupling of the scalar and gravitational fields. Using the reduction of $\vec{\alpha}_{2}$ and $\xi_{0}$ one has

$$
\mu \frac{d \lambda_{2}^{(i)}}{d \mu}=\sum_{j=1}^{3} \lambda_{2}^{(j)} \lambda_{0} L_{2}^{(i, j)}+L_{2}^{(i)} \alpha_{1}^{2} .
$$

If one does not consider additional free parameters (minimal reduction) then one has to be able to express $\lambda_{2}^{(i)}$ in terms of $\lambda_{0}, \alpha_{1}$ and $\alpha_{2}$. Consistency with the renormalization group equations leads to 


$$
\vec{\lambda}_{2}=\frac{\vec{l}_{2}}{\lambda_{0}} \alpha_{1}^{2}
$$

where $\overrightarrow{l_{2}}$ are power expansions in $\lambda_{0}$ with coefficients determined order by order as the solution of consistency equations which reduce to a linear system of equations.

If one inserts the reduction of parameters in the previous steps into the renormalization group equations (2.21) for the nonminimal couplings of dimension 6 , one obtains

$$
\mu \frac{d \xi_{2}^{(i)}}{d \mu}=\sum_{j=1}^{3} \xi_{2}^{(j)} \lambda_{0} X_{2}^{(i, j)}+\frac{X_{2}^{(i)}}{\lambda_{0}} \alpha_{1}^{2}
$$

Once more the reduction of the parameters $\xi_{2}^{(i)}$ is uniquely determined by the renormalization group equations,

$$
\vec{\xi}_{2}=\frac{\vec{x}_{2}}{\lambda_{0}^{2}} \alpha_{1}^{2}
$$

where each term in the expansion in powers of $\lambda_{0}$ of $\vec{x}_{2}$ is the solution of a linear system of equations.

The iterative procedure to reduce parameters can be applied to the remaining parameters of the effective theory. For the terms of order $\frac{1}{M^{4}}$ in $\mathcal{L}_{g}$ one has

$$
\mu \frac{d \vec{\alpha}_{4}}{d \mu}=\vec{A}_{4}^{(0)} \alpha_{1}^{2} \alpha_{2}+\frac{\vec{A}_{4}^{(1)}}{\lambda_{0}^{2}} \alpha_{1}^{4}
$$

and a reduction consistent with the renormalization group leads to

$$
\vec{\alpha}_{4}=\vec{a}_{4}^{(0)} \alpha_{2}^{2}+\frac{\vec{a}_{4}^{(1)}}{\lambda_{0}} \alpha_{1}^{2} \alpha_{2}+\frac{\vec{a}_{4}^{(2)}}{\lambda_{0}^{3}} \alpha_{1}^{4}
$$

The coefficients $\vec{a}_{4}^{(k)}, k=0,1,2$, are power expansions in $\lambda_{0}$ determined by a system of linear equations with one exception, the coefficient of $\lambda_{0}^{3}$ in $\vec{a}_{4}^{(2)}$, which does not appear in the consistency equations. Then once more the relations (3.10) are not a real reduction but a reparametrization in terms of the arbitrary constant coefficients of $\alpha_{1}^{4}$.

For the $\frac{1}{M^{4}}$ terms in $\mathcal{L}_{m}$ one has the reduced renormalization group equations

$$
\mu \frac{d \lambda_{4}^{(i)}}{d \mu}=\sum_{j} \lambda_{4}^{(j)} \lambda_{0} L_{4}^{(i, j)}+L_{4}^{(i)} \alpha_{1}^{2} \alpha_{2}+\frac{L_{4}^{\prime(i)}}{\lambda_{0}^{2}} \alpha_{1}^{4}
$$


and the reduction relations

$$
\vec{\lambda}_{4}=\frac{\vec{l}_{4}^{(0)}}{\lambda_{0}} \alpha_{1}^{2} \alpha_{2}+\frac{\vec{l}_{4}^{(1)}}{\lambda_{0}^{3}} \alpha_{1}^{4}
$$

which can be used to extend the reduction to the non-minimal couplings

$$
\vec{\xi}_{4}=\frac{\vec{x}_{4}^{(0)}}{\lambda_{0}^{2}} \alpha_{1}^{2} \alpha_{2}+\frac{\vec{x}_{4}^{(1)}}{\lambda_{0}^{4}} \alpha_{1}^{4} .
$$

The steps followed in the determination of the reduction of the parameters corresponding to terms of order $\frac{1}{M^{4}}$ can be repeated order by order in the expansion in $\frac{1}{M}$ to get the minimal reduction of the field theory of gravity coupled to a scalar field which is given by the relations

$$
\begin{aligned}
\vec{\alpha}_{2 n} & =\vec{a}_{2 n} \alpha_{2}^{n}\left[1+\mathcal{O}\left(\frac{\alpha_{1}^{2}}{\alpha_{2}}\right)\right] \\
\vec{\lambda}_{2 n} & =\frac{\vec{l}_{2 n}}{\lambda_{0}} \alpha_{2}^{n-1} \alpha_{1}^{2}\left[1+\mathcal{O}\left(\frac{\alpha_{1}^{2}}{\alpha_{2}}\right)\right] \\
\vec{\xi}_{2 n} & =\frac{\vec{x}_{2 n}}{\lambda_{0}^{2}} \alpha_{2}^{n-1} \alpha_{1}^{2}\left[1+\mathcal{O}\left(\frac{\alpha_{1}^{2}}{\alpha_{2}}\right)\right]
\end{aligned}
$$

The condition to have a real reduction of couplings is that $\alpha_{1}^{2} \ll \alpha_{2}$. In this case we have shown that there is a unique way to find perturbatively a unique lagrangian with the property that all the couplings can be written, at any renormalization scale $\mu$, in terms of three independent dimensionless parameters $\lambda_{0}, \alpha_{1}$ and $\alpha_{2}$. The reduction equations (3.14) as well as the $\mu$-dependence of the independent parameters (2.16), (2.18), (3.3) can be determined from a perturbative calculation of counterterms.

Each of the parameters of the reduced theory allows to identify a typical scale of the system. The scale associated to the parameter $\lambda_{0}$ can be taken for example as the scale $M_{0}$ such that $\frac{\lambda_{0}\left(\mu=M_{0}\right)}{4 \pi}=1$ and it fixes the range of validity of the perturbative approach to the effective theory. If we choose the scale $M$ in the energy expansion of the effective theory as $M_{0}$ then the second parameter $\alpha_{1}$ is associated to the scale characteristic of the classical limit, the Planck mass $M_{P l}$ through the relation $\frac{\alpha_{1}^{2}}{16 \pi}=\frac{M_{0}^{2}}{M_{P l}^{2}}$. The parameter $\alpha_{2}\left(M_{0}\right)$ allows to introduce a third scale in the system which is the scale characteristic of the fluctuations of the geometry $M_{R}$ generated by the matter system at the scale $M_{0}$. From the expansion 
of $\mathcal{L}_{g}$ in powers of the Riemann tensor one gets $\alpha_{2}\left(M_{0}\right)=\frac{M_{0}^{2}}{M_{R}^{2}}$ and the condition for the validity of the reduction $\alpha_{1}^{2} \ll \alpha_{2}$ in terms of the three scales of the system corresponds to the condition that $M_{R} \ll M_{P l}$.

\section{Non-minimal reduction}

The minimal reduction of couplings is an extension of the reduction found in the case of pure gravity in [8] with the addition of the self-coupling $\lambda_{0}$ to the independent parameters $\alpha_{1}$ and $\alpha_{2}$. An alternative to this reduction corresponds to introduce still another independent parameter from the matter system lagrangian. This can be done by going back to the renormalization group equations for $\lambda_{2}^{(i)}$ (3.5) and introducing a new independent parameter $\lambda_{2}$ with a renormalization scale dependence given by

$$
\mu \frac{d \lambda_{2}}{d \mu}=L_{2} \lambda_{0} \lambda_{2}
$$

where $L_{2}$ is a new expansion in powers of $\lambda_{0}$ to be conveniently choosen in a way compatible with the renormalization group equations. The most general form for the reduction of the parameters $\lambda_{2}^{(i)}$ compatible with dimensional arguments has three terms proportional to $\lambda_{2}, \alpha_{2}$ and $\alpha_{1}^{2}$ respectively with coefficients, depending on $\lambda_{0}$. These coefficients can be determined perturbatively through the consistency conditions on the reduction imposed by the renormalization group equations. The solution can be written in the form

$$
\lambda_{2}^{(i)}=l_{2,0}^{(i)} \lambda_{2}+\frac{l_{2,1}^{(i)}}{\lambda_{0}} \alpha_{1}^{2} .
$$

The parameter $\lambda_{2}$ can be chosen such that $L_{2}$ is a constant corresponding to one of the eigenvalues of the matrix given by the zero order term in the expansion of $L_{2}^{(i, j)}$ appearing in the renormalization group equation of $\lambda_{2}^{(i)}$ (3.5). The zero order terms in the expansion in powers of $\lambda_{0}$ of $l_{2,0}^{(i)}$ are the components of the corresponding eigenvector. The remaining terms in the expansion of the coefficients in the reduction, $l_{2,0}^{(i)}$ and $l_{2,1}^{(i)}$, are the solutions of the remaining consistency conditions which reduce to a system of linear equations. 
Once the new independent parameter $\lambda_{2}$ has been introduced, with its corresponding renormalization group equation (3.15), there is no difficulty to repeat the step by step reduction of the remaining dimensionless parameters in the effective theory. The results for the first terms in the $\frac{1}{M}$ expansion are

$$
\begin{aligned}
& \vec{\xi}_{2}=\frac{\vec{x}_{2,0}}{\lambda_{0}} \lambda_{2}+\frac{\vec{x}_{2,1}}{\lambda_{0}^{2}} \alpha_{1}^{2}, \\
& \vec{\alpha}_{4}=\vec{a}_{4,0} \alpha_{2}^{2}+\frac{\vec{a}_{4,1}}{\lambda_{0}} \alpha_{2} \alpha_{1}^{2}+\frac{\vec{a}_{4,2}}{\lambda_{0}^{2}} \lambda_{2} \alpha_{1}^{2}+\frac{\vec{a}_{4,3}}{\lambda_{0}^{3}} \alpha_{1}^{4}, \\
& \vec{\lambda}_{4}=\frac{\vec{l}_{4,0}}{\lambda_{0}} \lambda_{2}^{2}+\frac{\vec{l}_{4,1}}{\lambda_{0}^{2}} \lambda_{2} \alpha_{1}^{2}+\frac{\vec{l}_{4,2}}{\lambda_{0}} \alpha_{2} \alpha_{1}^{2}+\frac{\vec{l}_{4,3}}{\lambda_{0}^{3}} \alpha_{1}^{4}, \\
& \vec{\xi}_{4}=\frac{\vec{x}_{4,0}}{\lambda_{0}^{2}} \lambda_{2}^{2}+\frac{\vec{x}_{4,1}}{\lambda_{0}^{3}} \lambda_{2} \alpha_{1}^{2}+\frac{\vec{x}_{4,2}}{\lambda_{0}^{2}} \alpha_{2} \alpha_{1}^{2}+\frac{\vec{x}_{4,3}}{\lambda_{0}^{4}} \alpha_{1}^{4} .
\end{aligned}
$$

The expansion in powers of $\lambda_{0}$ of the reduction coefficients $\vec{a}, \vec{l}, \vec{x}$, is fixed by a set of linear systems of equations with the exception of the term proportional to $\lambda_{0}^{3}$ in $\vec{a}_{4,3}$ which remains undetermined. Once more a real reduction of couplings requires to assume that $\alpha_{1}^{2} \ll \alpha_{2}$ and to neglect terms supressed by powers of $\frac{\alpha_{1}^{2}}{\alpha_{2}}$ in the reduction equations (3.17).

The new independent parameter $\lambda_{2}$ in this nonminimal reduction corresponds to a new mass scale $M_{E}$ in the energy expansion of the matter system related to the dimensionless

parameter $\lambda_{2}$ by $\lambda_{2}\left(M_{0}\right)=\frac{M_{0}^{2}}{M_{E}^{2}}$. One can see the nonminimal reduction as an extension of the scalar effective field theory with two free parameters $\lambda_{0}$ and $\lambda_{2}$. The gravitational interaction introduces at least two additional parameters $\alpha_{1}$ and $\alpha_{2}$. The main difference with respect to the minimal reduction discussed in the previous subsection is that the contributions of higher dimensional operators in the matter system lagrangian are not necessarily suppressed by inverse powers of the Planck mass.

\section{MASSIVE CASE. COSMOLOGICAL CONSTANT PROBLEM}

If one considers the gravitational interaction of a massive spinless particle then one has to include a term $\lambda_{-2} M^{2} \phi^{2}$ in the matter lagrangian density. The dimensionless parameter $\lambda_{-2}$ has to be taken into account in the discussion based on dimensional arguments leading to 
the general structure of the renormalization group equations. The homogeneity conditions of the $\beta$ functions for the parameters $\alpha_{1}, \vec{\alpha}_{2 n}, \vec{\lambda}_{2 n}, \vec{\xi}_{2 n}$ include the rescaling of the additional parameter, $\lambda_{-2}^{\prime}=t^{-2} \lambda_{-2}$, and the simple triangular structure is lost due to the contributions proportional to positive powers of $\lambda_{-2}$ which will be accompanied by the parameters corresponding to terms of higher dimensionality. If one wants the reduction of couplings to be applicable also in this case then one has to assume that the dimensionless parameter $\lambda_{-2}$ is sufficiently small to treat its effects as a small perturbation. The additional parameter is associated to a new mass scale in the system, the scalar particle mass $m^{2}=\lambda_{-2} M^{2}$, and the reduction of couplings requires this scale to be much smaller than any of the other mass scales of the system. The reduction of couplings identified in the previous section for the massless case can be taken as the zero order term of an expansion of the reduction equations in powers of the parameter $\lambda_{-2}$.

Another effect of the mass of the scalar particle is that one has to consider simultaneously a cosmological constant. This can be seen from the renormalization group equation for the dimensionless parameter $\alpha_{-2}$, corresponding to the cosmological term, which can be written in the form

$$
\mu \frac{d \alpha_{-2}}{d \mu}=\lambda_{-2}^{2} \alpha_{1}^{2} \hat{\beta}_{\alpha_{-2}}\left(\lambda_{-2}, \vec{\alpha}, \vec{\lambda}, \vec{\xi}\right)+\mathcal{O}\left(\alpha_{-2}\right)
$$

Dimensional arguments and the fact that all the couplings of the scalar field to the gravitational field $h_{\mu \nu}$ are proportional to $\alpha_{1}$ have been used to write the contribution to the $\beta$ function of the dimensionless cosmological parameter due to the remaining parameters in terms of the function $\hat{\beta}_{\alpha_{-2}}$ which satisfies the homogeneity condition

$$
\hat{\beta}_{\alpha_{-2}}\left(\vec{\alpha}^{\prime}, \vec{\lambda}^{\prime}, \vec{\xi}^{\prime}\right)=\hat{\beta}_{\alpha_{-2}}(\vec{\alpha}, \vec{\lambda}, \vec{\xi})
$$

The first terms in the expansion in powers of $\lambda_{-2}$ for the renormalization group equation of the dimensionless cosmological parameter are

$$
\mu \frac{d \alpha_{-2}}{d \mu}=\lambda_{-2}^{2} \alpha_{1}^{2}\left\{B_{-2}^{(1)}+\left[\sum_{i} B_{-2}^{(i, 1)} \lambda_{2}^{(i)}+\sum_{i} B_{-2}^{(i, 2)} \xi_{2}^{(i)}+B_{-2}^{(2)} \alpha_{1}^{2}\right] \lambda_{-2}+\right.
$$




$$
\begin{aligned}
& {\left[\sum_{i} B_{-2}^{(i, 3)} \lambda_{4}^{(i)}+\sum_{i} B_{-2}^{(i, 4)} \xi_{4}^{(i)}+\sum_{i, j} B_{-2}^{(i, j, 1)} \lambda_{2}^{(i)} \lambda_{2}^{(j)}+\right.} \\
& \sum_{i, j} B_{-2}^{(i, j, 2)} \lambda_{2}^{(i)} \xi_{2}^{(j)}+\sum_{i, j} B_{-2}^{(i, j, 3)} \xi_{2}^{(i)} \xi_{2}^{(j)}+\sum_{i} B_{-2}^{(i, 5)} \alpha_{2}^{(i)} \alpha_{1}^{2}+ \\
& \left.\sum_{i} B_{-2}^{(i, 6)} \lambda_{2}^{(i)} \alpha_{1}^{2}+\sum_{i} B_{-2}^{(i, 7)} \xi_{2}^{(i)} \alpha_{1}^{2}+B_{-2}^{(3)} \alpha_{1}^{4}\right] \lambda_{-2}^{2}+ \\
& \left.\mathcal{O}\left(\lambda_{-2}^{3}\right)\right\}+\mathcal{O}\left(\alpha_{-2}\right) .
\end{aligned}
$$

The cosmological constant parameter $\Lambda=\frac{\alpha_{-2}}{\alpha_{1}^{2}} M^{4}$ can be written in units of the scalar mass by using the dimensionless parameters $\alpha_{1}, \alpha_{-2}$ and $\lambda_{-2}$ as

$$
\frac{\Lambda}{m^{4}}=\frac{\alpha_{-2}}{\alpha_{1}^{2} \lambda_{-2}^{2}}=\frac{\alpha_{-2}}{\lambda_{-2}} \frac{1}{\alpha_{1}^{2} \lambda_{-2}} .
$$

This, together with the renormalization group equation (4.3), gives a reformulation of the cosmological constant problem at the level of the effective field theoretic formulation. It is not possible to have $\alpha_{-2} \ll \alpha_{1}^{2} \lambda_{-2}{ }^{2}$ over the energy range of validity of the effective theory and then it is not possible to have $\frac{\Lambda}{m^{4}} \ll 1$ as required by the experimental upper bound for the cosmological constant.

In order to discuss possible solutions to the cosmological constant problem one has first to consider, instead of a scalar field, a realistic matter system including fermion and gauge fields with the strong and electroweak interactions of the minimal standard model of particle physics. If the matter system content of fields and interactions is such that as a consequence of a symmetry $\hat{\beta}_{\alpha_{-2}}=0$, then

$$
\mu \frac{d \alpha_{-2}}{d \mu} \propto \alpha_{-2}
$$

and the cosmological parameter can be made arbitrarily small $\mathrm{f}_{\text {. }}$

Taking into account that $\alpha_{1}^{2} \lambda_{-2}=\frac{m^{2}}{M_{P l}^{2}}$ is of the order of $10^{-32}$ for a mass scale of the matter system $m$ of the order of 1 Tev, another possibility to solve the cosmological problem

$\ddagger$ A supersymmetric extension of the minimal standard model is a candidate but it is not clear how the required soft supersymmetry breaking terms can be made compatible with the vanishing of $\hat{\beta}_{\alpha_{-2}}$. 
is based on the assumption that the first two terms in the expansion of $\beta_{\alpha_{-2}}$ vanish. The remaining terms can be made compatible with the smallness of the cosmological constant if the combinations of parameters, appearing in the coefficient of $\lambda_{-2}{ }^{2}$ inside the brackets in (4.1), are not much bigger than $\alpha_{1}^{4}$.

If one considers a minimal reduction of the effective theory for a realistic matter system with the same number of independent parameters $\lambda_{-2}, \lambda_{0}, \alpha_{1}, \alpha_{2}$ as in the case of a scalar field, then the renormalization group equation of the cosmological parameter will be given by

$$
\begin{array}{r}
\mu \frac{d \alpha_{-2}}{d \mu}=\lambda_{-2}^{2} \alpha_{1}^{2}\left\{A_{-2}^{(1)}+A_{-2}^{(2)} \alpha_{1}^{2} \lambda_{-2}+A_{-2}^{(3)} \alpha_{1}^{2} \alpha_{2} \lambda_{-2}^{2}+\right. \\
\left.A_{-2}^{(4)} \alpha_{1}^{4} \lambda_{-2}^{2}+\mathcal{O}\left(\lambda_{-2}^{3}\right)\right\}+\mathcal{O}\left(\alpha_{-2}\right)
\end{array}
$$

where the coefficients $A_{-2}^{(k)}$ are power expansions in the parameter $\lambda_{0}$. In this case the possible solution of the cosmological constant problem based on the vanishing of the first few terms in the $\lambda_{-2}$ expansion of the $\beta$-function gives just two conditions on the matter system,

$$
A_{-2}^{(1)}=0 \quad A_{-2}^{(2)}=0
$$

Assuming that the remaining coefficient expansions are of order one, one has the additional condition for the independent dimensionless parameters $\frac{\alpha_{2}}{\alpha_{1}^{2}} \leq 10^{6}$ which corresponds to the restriction on the mass scales of the effective theory $\frac{M_{R}^{2}}{M_{P l}^{2}} \geq 10^{-6}$. On the contrary, if the solution to the cosmological problem is based on some symmetry of the underlying theory leading to $A_{-2}^{(k)}=0$ there is no restriction, due to the smallness of the cosmological constant, on the mass scales of the effective theory.

The cosmological constant problem can be discussed along the same lines if there is a nonminimal reduction of the effective theory with an additional parameter $\lambda_{2}$. In this case one has the renormalization group equation for the dimensionless cosmological parameter

$$
\begin{aligned}
\mu \frac{d \alpha_{-2}}{d \mu}= & \lambda_{-2}{ }^{2} \alpha_{1}^{2}\left\{A_{-2}^{\prime}{ }^{(1)}+\left[A_{-2}^{\prime}{ }^{(2)} \alpha_{1}^{2}+A_{-2}^{\prime}{ }^{(3)} \lambda_{2}\right] \lambda_{-2}+\right. \\
& +\left[A_{-2}^{\prime}{ }^{(4)} \lambda_{2}^{2}+A_{-2}^{\prime}{ }^{(5)} \alpha_{2} \alpha_{1}^{2}+A_{-2}^{\prime}{ }^{(6)} \lambda_{2} \alpha_{1}^{2}+A_{-2}^{\prime}{ }^{(7)} \alpha_{1}^{4}\right] \lambda_{-2}{ }^{2}+ \\
& \left.+\mathcal{O}\left(\lambda_{-2}^{3}\right)\right\}+\mathcal{O}\left(\alpha_{-2}\right)
\end{aligned}
$$


In this case a solution to the cosmological constant problem based on the vanishing of the first two terms in the expansion in powers of $\lambda_{-2}$ requires that the matter system satisfies the three conditions

$$
A_{-2}^{\prime}{ }^{(k)}=0 \quad k=1,2,3
$$

and one also has the bounds for the parameters $\lambda_{2}$ and $\alpha_{2}$,

$$
\frac{\alpha_{2}}{\alpha_{1}^{2}} \leq 10^{6}, \frac{\lambda_{2}}{\alpha_{1}^{2}} \leq 10^{3}
$$

which gives a restriction on the aditional mass scale $M_{E}, \frac{M_{E}^{2}}{M_{P l}^{2}} \geq 10^{-3}$.

\section{SUMMARY}

It has been shown that the perturbative renormalization of the theory of a scalar field coupled to the gravitational field, which requires an infinite number of counterterms, is compatible with the presence of only a finite number of renormalized parameters which can be choosen arbitrarily at a given scale. In this sense one has a theory with the same predictibility as a renormalizable theory in the power counting sense. The corrections to the low energy limit due to the higher order terms in the energy expansion of the effective theory do not necessarily involve additional free parameters. This result found in the case of pure gravity [8] has been extended to the case of a scalar field coupled to gravity in two different forms (minimal and non-minimal reduction of couplings). The effective theory has several characteristic mass scales: the scale associated to the classical limit (the Planck

mass $M_{P l}$ ), the scale associated to the fluctuations of the geometry $\left(M_{R}\right)$, the scale of the fluctuations of the matter system $\left(M_{E}\right)$, the scale limiting the validity of the perturbative treatment $\left(M_{0}\right)$ and the mass of the scalar field $(m)$. Each of these scales is in one to one correspondence with the independent dimensionless parameters of the effective theory after a reduction of couplings compatible with the renormalization group equations. The reduction of couplings puts some limitations on the mass scales. The reduction of the couplings in the 
gravitational lagrangian requires $\frac{\alpha_{2}}{\alpha_{1}^{2}} \gg 1$ and then $M_{R} \ll M_{P l}$. The treatment of the scalar mass as a perturbation requires $\lambda_{-2} \lambda_{2} \ll 1$ which implies that $m \ll M_{E}$. In the case of minimal reduction one has $\lambda_{2} \sim \alpha_{1}^{2}$ and then $M_{E} \sim M_{P l}$.

In order to have an energy range within the perturbative domain where some of the low energy limit corrections have to be included, it is necessary for either $M_{R}$ or $M_{E}$ to be smaller than $M_{0}$. In the case of minimal reduction of couplings the contribution from a term $\mathcal{L}_{m}^{(n)}$ in the matter lagrangian, which is of order $\frac{\lambda_{2 n}}{M^{2 n}} E^{2 n}$ when one is considering the matter system at energies of order $E$, gives an effect of order $\left(\frac{E}{M_{P l}}\right)\left(\frac{E^{2}}{M_{R}^{2}}\right)^{n-1}$. Then even if the scale $M_{0}$ for the domain of validity of the perturbative treatment can be much lower than $M_{P l}$ one has to consider energies $E \sim M_{R}$ in order to go beyond the low energy limit.

It is natural to expect that the results found for a scalar field coupled to gravity can be generalized to more general cases for the matter system. Instead of the scalar self-coupling $\lambda_{0}$ one will have a set of dimensionless couplings for the non gravitational interactions and instead of the mass of the scalar one will have one or several masses for the matter fields.

The cosmological constant problem has been reformulated at the level of the renormalization group equations. In the presence of a mass for the scalar field the (logarithmic) derivative of the cosmological parameter with respect to the renormalization scale has a contribution which is not proportional to the cosmological parameter. Then it is not possible to have an arbitrarily small value for this parameter over a given range of scales. Two possible solutions of this problem have been considered corresponding to the possibility that these terms in the $\beta$-function of the cosmologic parameter which are not proportional to it either vanish for an appropriate choice of matter lagrangian or are sufficiently small to be compatible with the smallness of the cosmological constant. The number of conditions that the matter system have to satisfy, in order to have a solution to the cosmological constant problem based on the second possibility mentioned above, is significantly reduced if one considers an effective theory with a reduction of couplings.

\section{Acknowledgments}


This work was partially supported by the CICYT (proyecto AEN 94-0218). The work of M.A. has been supported by a DGA fellowship. 


\section{REFERENCES}

[1] S. Weinberg, "The Quantum Theory of Fields", Cambridge University Press, Cambridge 1995.

[2] F. J. Dyson, Phys. Rev. 75, (1949) 486,1736.

[3] For a recent discussion see,

J. Gomis and S. Weinberg, "Are Nonrenormalizable Gauge Theories Renormalizable ?", hep-th/9510087.

[4] S. Weinberg, Physica 96A, (1979) 327.

H. Georgi, "Weak Interactions and Modern Particle Theory", Benjamin Cummings, Menlo Park, 1984.

J. Gasser and H. Leutwyler, Nucl. Phys. B250, (1985) 465.

J. F. Donoghue, E. Golowich and B. R. Holstein, "Dynamics of the Standard Model", Cambridge University Press, Cambridge 1992.

[5] J. F. Donoghue, Phys.Rev.Lett. 72, (1994) 2996 ; Phys.Rev. D50, (1994) 3874.

[6] A very clear discussion on this point can be found in

A. V. Manohar, "Effective Field Theories", hep-ph/9508245.

[7] C. J. Isham, "Structural Issues in Quantum Gravity", gr-qc/9510063.

[8] M. Atance and J. L. Cortés, "Effective Field Theory of pure Gravity and the Renormalization Group", hep-th/9604076.

[9] for a review of the problem see

S. Weinberg, Rev.Mod.Phys. 61, (1989) 1.

[10] see for instance, 
D. J. Gross, Methods in Field Theory, Proceedings of the Les Houches Summer School, 1975, edited by R. Balian and J. Zinn-Justin, North-Holland, Amsterdam, 1976

[11] G. 't Hooft and M. Veltman, Nucl.Phys. B44, (1972) 189.

[12] G. 't Hooft, Nucl.Phys. B61, (1973) 455.

[13] G. 't Hooft and M. Veltman, Ann. Inst. H. Poincare A20, (1974) 69.

[14] M. Goroff and A. Sagnotti, Nucl.Phys. B266, (1986) 709.

[15] M. Veltman, Methods in Field Theory, Proceedings of the Les Houches Summer School, 1975, edited by R. Balian and J. Zinn-Justin, North-Holland, Amsterdam, 1976.

[16] R. E. Kallosh, O. V. Tarasov and I. V. Tyutin, Nucl.Phys, B137, (1978) 145.

[17] For a recent review with a list of references see R. Oehme, "Reduction of Coupling Parameters", hep-th/9511006.

[18] R. Oehme and W. Zimmerman, Commun.Math.Phys. 97, (1985) 569.

W. Zimmerman, Commun.Math.Phys. 97, (1985) 211.

[19] R. J. Perry and K. G. Wilson, Nucl.Phys. B403, (1993) 587. 